\title{
COMPACTNESS CRITERION FOR FRACTIONAL INTEGRATION OPERATOR OF INFINITESIMAL ORDER
}

\section{A.M. ABYLAYEVA, A.O. BAIARYSTANOV}

Abstract. We obtain necessary and sufficient conditions of compactness for the operator

$$
K f(x)=\int_{0}^{x} \ln \frac{x}{x-s} \frac{f(s)}{s} d s
$$

from $L_{p, v}$ in $L_{q, u}$ as $1<p \leqslant q<\infty$ and $v(x)=x^{-\gamma}, \gamma>0$, where $L_{q, u}$ is the set of all measurable on $(0, \infty)$ functions $f$ with finite norm $\|u f\|_{q}$.

Keywords: compactness, fractional integration operator, Riemann-Liouville operator, singular operator, adjoint operator, Holder inequality, weighted inequalities.

\section{INTRODUCTION}

Let $1<p \leqslant q<\infty, \frac{1}{p}+\frac{1}{p^{\prime}}=1, R_{+}=(0, \infty), u, v: R_{+} \rightarrow R$ be weight functions, i.e., nonnegative and measurable on $R_{+}$.

Starting from 70s of the last century, in the world mathematical literature a weighted estimate

$$
\|u K f\|_{q} \leqslant C\|v f\|_{p}
$$

is intensively studied for various classes of operators $K$, where $\|\cdot\|_{p}$ is the usual norm of the space $L_{p} \equiv L_{p}(R)$. In what follows by $L_{p, v}$ we indicate the set of the functions $f: R_{+} \rightarrow R$ with finite norm $\|f\|_{p, v}=\|v f\|_{p}$. The survey of the studies of estimate (1) between 1970 and 1982 was presented in [1]. Some directions of studies of estimates (1) performed for integral operators before 2003 were provided in [2]. In paper [3] a sequence of the classes of nonnegative functions $K(\cdot, \cdot)$ was given as well as the complete description of the weights $u$ and $v$ for which the integral operator

$$
K f(x)=\int_{0}^{x} K(x, s) f(s) d s
$$

obeys (1) if its kernel belongs to these classes. However, these results do not include operator (2) if its kernel $K(\cdot, \cdot)$ has a singularity, for instance, Riemann-Liouville operator

$$
R_{\alpha} f(x)=\int_{0}^{x} \frac{f(s) d s}{(x-s)^{1-\alpha}}
$$

as $0<\alpha<1$. Estimate (1) for operator (3) in the general case is still an open question. Nevertheless, the following cases were studied, $u \equiv v$ in $[4], v \equiv 1$ in $[5,6]$, and the case of non-increasing of one of weight functions $u, v$ in [7].

A.M. Abylayeva, A.O. Baiarystanov, Compactness Criterion for fractional integration opERATOR OF INFINITESIMAL ORDER.

(C) Abylayeva A.M., Baiarystanov A.O. 2013.

Submitted December 23, 2011. 
The operator of the form

$$
K f(x)=\int_{0}^{x} \ln \frac{x}{x-s} \frac{f(s)}{s} d s
$$

is called a fractional integration operator of infinitesimal order (see [8], p. 34).

In [9] estimate (1) for operator (4) was studied in the case $v(x)=x^{-\gamma}, \gamma>0$. It is equivalent to the estimate

$$
\left\|u T_{\gamma} f\right\|_{q} \leqslant C\|f\|_{p}
$$

for the operator

$$
T_{\gamma} f(x)=\int_{0}^{x} s^{\gamma-1} \ln \frac{x}{x-s} f(s) d s
$$

Since

$$
\ln \frac{x}{x-s}=\int_{0}^{s} \frac{d t}{x-t} \quad \text { for } x>s \geq 0
$$

the inequality

$$
\frac{s}{x-s}>\ln \frac{x}{x-s}>\frac{s}{x}, \quad x>s>0
$$

holds true. The function $\ln \frac{x}{x-s}$ decays w.r.t. $x$ and increases w.r.t. $s$ as $x>s \geq 0$, and the functions $x \ln \frac{x}{x-s}, \frac{1}{s} \ln \frac{x}{x-s}$ decay w.r.t. $x$ and decrease w.r.t. $s$ as $x>s>0$. Indeed,

$$
\begin{gathered}
\frac{\partial}{\partial x}\left(x \ln \frac{x}{x-s}\right)=\ln \frac{x}{x-s}-\frac{s}{x-s}<0, \\
\frac{\partial}{\partial s}\left(\frac{1}{s} \ln \frac{x}{x-s}\right)=\frac{1}{s^{2}}\left(\frac{s}{x-s}-\ln \frac{x}{x-s}\right)>0
\end{gathered}
$$

as $x>s>0$. We observe that for a differentiable function $f$ estimate (1) for operator (4) is equivalent to the inequality

$$
\left(\int_{0}^{\infty}\left|u(x) \int_{0}^{x} \frac{f(x)-f(s)}{x-s} d s\right|^{q} d x\right)^{\frac{1}{q}} \leqslant C\left(\int_{0}^{\infty}\left|f^{\prime}(x) x^{1-\gamma}\right|^{p} d x\right)^{\frac{1}{p}}
$$

In the paper we assume the following. The indeterminate forms $0 \cdot \infty, \frac{0}{0}, \frac{\infty}{\infty}$ are assumed to be zero. The inequality of the form $A \leqslant \beta B$, where a positive constant $\beta$ can depend on parameters $p, q$, and $\gamma$, will be written as $A \ll B$, while the relation $A \approx B$ will be indicated as $A \ll B \ll A$.

Let $\chi_{(a, b)}(\cdot)$ be the characteristic function of the interval $(a, b), Z$ is the set of integers.

In work [9] the authors obtained the criteria of boundedness for the operator $T_{\gamma}$ and its adjoint

$$
T_{\gamma}^{*} g(s)=s^{\gamma-1} \int_{s}^{\infty} g(x) \ln \frac{x}{x-s} d x
$$

acting from $L_{p}$ into $L_{q, u}$.

In particular, the following theorems were proven. 
Theorem A. Let $1<p \leqslant q<\infty, \gamma>\frac{1}{p}$. The operator $T_{\gamma}$ is bounded as that from $L_{p}$ in $L_{q, u}$ if and only if

$$
D_{\gamma}=\sup _{x>0} D_{\gamma}(x)<\infty, \text { where } D_{\gamma}(x)=x^{\gamma+\frac{1}{p^{\prime}}}\left(\int_{x}^{\infty} t^{-q} u(t) d t\right)^{\frac{1}{q}} .
$$

At that, $\left\|T_{\gamma}\right\| \approx D_{\gamma}$.

Theorem B. Let $1<p \leqslant q<\infty, \gamma>1-\frac{1}{q}$. Then the operator $T_{\gamma}^{*}$ is bounded as that from $L_{p, v}$ into $L_{q}$ if and only if

$$
D_{\gamma}^{*}=\sup _{x>0} D_{\gamma}^{*}(x) \equiv \sup _{x>0} x^{\gamma+\frac{1}{q}}\left(\int_{x}^{\infty} t^{-p^{\prime}} v^{1-p^{\prime}}(t) d t\right)^{\frac{1}{p^{\prime}}}<\infty .
$$

At that, $\left\|T_{\gamma}^{*}\right\| \approx D_{\gamma}^{*}$.

In the present work we study the compactness of the operator $T_{\gamma}$ acting from $L_{p}$ into $L_{q, u}$.

\section{MAIN RESULT}

Theorem 1. Let $1<p \leqslant q<\infty, \gamma>\frac{1}{p}$. The operator $T_{\gamma}$ is compact from $L_{p}$ in $L_{q, u}$ if and only if $D_{\gamma}<\infty$ and

$$
\lim _{x \rightarrow 0} D_{\gamma}(x)=\lim _{x \rightarrow \infty} D_{\gamma}(x)=0 .
$$

Proof. Necessity. Let $T_{\gamma}$ be a compact operator from $L_{p}$ into $L_{q, u}$. By Theorem A $D_{\gamma}<\infty$.

Let us prove the validity of the conditions (9). For $0<s<\infty$ we consider a family of the functions

$$
f_{s}(x)=\chi_{(0, s)}(x) s^{-\frac{1}{p}}, x>0
$$

with norm

$$
\|f\|_{L_{p}}=\left(\int_{0}^{\infty}\left|f_{s}(x)\right|^{p} d x\right)^{\frac{1}{p}}=\left(\int_{0}^{s} s^{-1} d x\right)^{\frac{1}{p}}=s^{-\frac{1}{p}}\left(\int_{0}^{s} d x\right)^{\frac{1}{p}}=1 .
$$

Let us show that family of the functions (10) converges weakly to zero in $L_{p}$. By Theorem [10] on the general form of linear continuous functionals in a Lebesgue space, the linear continuous functional in $L_{p}$ reads as

$$
\int_{0}^{\infty} f(x) g(x) d x, \text { where } g \in L_{p^{\prime}}
$$

Employing Hölder inequality, we deduce

$$
\begin{gathered}
\int_{0}^{\infty} f_{s}(x) g(x) d x=\int_{0}^{s} s^{-\frac{1}{p}} g(x) d x \leqslant \\
\leqslant s^{-\frac{1}{p}}\left(\int_{0}^{s} d x\right)^{\frac{1}{p}}\left(\int_{0}^{s}|g(x)|^{p^{\prime}} d x\right)^{\frac{1}{p^{\prime}}}=\left(\int_{0}^{s}|g(x)|^{p^{\prime}} d x\right)^{\frac{1}{p^{\prime}}} .
\end{gathered}
$$


For each $g \in L_{p^{\prime}}$ the latter integral in (12) tends to zero as $s \rightarrow 0$ that implies the weak convergence $f_{s} \rightarrow 0$ in $L_{p}$ as $s \rightarrow 0$. Then by the properties of compact operators in a Banach space

$$
\lim _{s \rightarrow 0}\left\|T_{\gamma} f_{s}\right\|_{q, u}=0 .
$$

Since $\ln \frac{x}{x-t} \geq \frac{t}{x}$ as $0<t<x$, we have

$$
\begin{gathered}
\left\|T_{\gamma} f_{s}\right\|_{q, u}=\left(\int_{0}^{\infty} u(x)\left|\int_{0}^{x} t^{\gamma-1} \ln \frac{x}{x-t} f_{s}(t) d t\right|^{q} d x\right)^{\frac{1}{q}} \geq \\
\geq\left(\int_{s}^{\infty} u(x)\left|\int_{0}^{s} t^{\gamma-1} s^{-\frac{1}{p}} \ln \frac{x}{x-t} d t\right|^{q} d x\right)^{\frac{1}{q}} \geq \\
\geq s^{-\frac{1}{p}}\left(\int_{0}^{s} t^{\gamma} d t\right)\left(\int_{s}^{\infty} x^{-q} u(x)\right)^{\frac{1}{q}}=\frac{1}{\gamma+1} D_{\gamma}(s) .
\end{gathered}
$$

It follows from (13) and (14) that $\lim _{s \rightarrow 0} D_{\gamma}(s)=0$, i.e., the first relation in (9). Let us show that the second relation in (9) holds true. The compactness of the operator $T_{\gamma}$ yields the same for adjoint operator $T_{\gamma}^{*}(8)$ acting from $L_{q^{\prime}, u^{1-q^{\prime}}}$ into $L_{p^{\prime}}$. For $0<s<\infty$ we introduce the family of the functions

$$
g_{s}(x)=\chi_{(s, \infty)}(x)\left(\int_{s}^{\infty} t^{-q} u(t) d t\right)^{-\frac{1}{q^{\prime}}} u(x) x^{1-q} .
$$

The conditions $D_{\gamma}<\infty$ implies that the integral in (15) is convergent. Let us show that $g_{s} \in L_{q^{\prime}, u^{1-q^{\prime}}}$ for each $s>0$.

Indeed,

$$
\begin{gathered}
\left\|g_{s}\right\|_{q^{\prime}, u^{1-q^{\prime}}}=\left(\int_{0}^{\infty}\left|g_{s} x\right|^{q^{\prime}} u^{1-q^{\prime}}(x) d x\right)^{\frac{1}{q^{\prime}}}= \\
=\left(\int_{s}^{\infty} t^{-q} u(t) d t\right)^{-\frac{1}{q^{\prime}}}\left(\int_{s}^{\infty}\left(u(x) x^{1-q}\right)^{q^{\prime}} u^{1-q^{\prime}}(x) d x\right)^{\frac{1}{q^{\prime}}}=1 .
\end{gathered}
$$

By (16) for all $f \in L_{q, u}$

$$
\begin{gathered}
\int_{0}^{\infty} g_{s}(x) f(x) d x=\int_{s}^{\infty} g_{s}(x) f(x) d x \leqslant\left(\int_{s}^{\infty}\left|g_{s}(x)\right|^{q^{\prime}} u^{1-q^{\prime}}(x) d x\right)^{\frac{1}{q^{\prime}}} \times \\
\times\left(\int_{s}^{\infty}|f(x)|^{q} u(x) d x\right)^{\frac{1}{q}}=\left(\int_{s}^{\infty}|f(x)|^{q} u(x) d x\right)^{\frac{1}{q}} .
\end{gathered}
$$

Passing in the latter inequality to the limit as $s \rightarrow \infty$, we see that $g_{s} \rightarrow 0$ weakly in $L_{q^{\prime}, u^{1-q^{\prime}}}$ as $s \rightarrow \infty$. Hence, $T_{\gamma}^{*} g_{s}$ (by compactness of $T_{\gamma}^{*}$ ) converges to zero as $s \rightarrow \infty$ in the sense of $L_{p^{\prime}}$-norm, i.e.,

$$
\lim _{s \rightarrow \infty}\left\|T_{\gamma}^{*} g_{s}\right\|_{p^{\prime}}=0
$$


We have

$$
\begin{gathered}
\left\|T_{\gamma}^{*} g_{s}\right\|_{p^{\prime}}=\left(\int_{0}^{\infty}\left|t^{\gamma-1} \int_{t}^{\infty} g_{s}(x) \ln \frac{x}{x-t} d x\right|^{p^{\prime}} d t\right)^{\frac{1}{p^{\prime}}} \geq \\
\geq\left(\int_{0}^{s} t^{p^{\prime}(\gamma-1)}\left(\int_{s}^{\infty} \frac{u(x)}{x^{q-1}} \ln \frac{x}{x-t} d x\right)^{p^{\prime}} d t\right)^{\frac{1}{p^{\prime}}}\left(\int_{s}^{\infty} x^{-q} u(x) d x\right)^{-\frac{1}{q^{\prime}}} \geq
\end{gathered}
$$

(we again employ inequality $\ln \frac{x}{x-t} \geq \frac{t}{x}$ )

$$
\geq\left(\int_{s}^{\infty} x^{-q} u(x) d x\right)^{\frac{1}{q}}\left(\int_{0}^{s} t^{p^{\prime}(\gamma-1)} t^{p^{\prime}} d t\right)^{\frac{1}{p^{\prime}}}=\left(\frac{1}{p^{\prime} \gamma+1}\right)^{\frac{1}{p^{\prime}}} D_{\gamma}(s) .
$$

Together with (17) it implies the second relation in (9). The proof of the necessity is completed. Sufficiency. Let $0<a<b<\infty$ and

$$
P_{a} f=\chi_{(0, a)} f, \quad P_{a b} f=\chi_{[a, b)} f, \quad Q_{b} f=\chi_{[b, \infty)} f .
$$

Then for the operator $T_{\gamma}$

$$
T_{\gamma} f=P_{a b} T_{\gamma} P_{a b}+P_{a} T_{\gamma} P_{a} f+P_{a b} T_{\gamma} P_{a} f+Q_{b} T_{\gamma} f .
$$

Let us show that the operator $P_{a b} T_{\gamma} P_{a b}$ is compact from $L_{p}$ into $L_{q, u}$. Since $P_{a b} T_{\gamma} P_{a b} f(x)=P_{a b} T_{\gamma} \chi_{[a b)}(x) f(x)=0$ for $x \notin[a, b)$, it is sufficient to show that the operator $P_{a b} T_{\gamma} P_{a b}$ is compact from $L_{p}(a, b)$ into $L_{q, u}(a, b)$, and, in its turn, it is equivalent to the compactness of the operator $T f(x)=\int_{a}^{b} K(x, s) f(s) d s$ with kernel $K(x, s)=u^{\frac{1}{q}}(x) \chi_{(a, b)}(x-s) s^{\gamma-1} \ln \frac{x}{x-s}$ from $L_{p}(a, b)$ into $L_{q}(a, b)$ that by the local integrability of the function $u$ satisfies the condition

$$
\int_{a}^{b}\left(\int_{a}^{b}|K(x, s)|^{p^{\prime}} d s\right)^{\frac{q}{p^{\prime}}} d x=\int_{a}^{b} u(x)\left(\int_{a}^{x}\left(s^{\gamma-1} \ln \frac{x}{x-s}\right)^{p^{\prime}} d s\right)^{\frac{q}{p^{\prime}}} \leqslant
$$

(we employ the inequality $\frac{s}{x-s} \geq \ln \frac{x}{x-s}$ as $x>s>0$ )

$$
\leqslant \int_{a}^{b} u(x)\left(\int_{a}^{x} s^{p^{\prime} \gamma}\left(\frac{1}{x-s}\right)^{p^{\prime}} d s\right)^{\frac{q}{p^{\prime}}} \leqslant\left(\int_{a}^{b} s^{p^{\prime} \gamma} d s\right)^{\frac{q}{p^{\prime}}} \int_{a}^{b} u(x) x^{-q} d x<\infty .
$$

Therefore, by Kantorovich test [10], the operator $T_{\gamma}$ is compact from $L_{p}(a, b)$ into $L_{q}(a, b)$, that is equivalent to the compactness from $L_{p}$ into $L_{q, u}$ of the operator $P_{a b} T_{\gamma} P_{a b}$. It follows from (18) that

$$
\left\|T_{\gamma}-P_{a b} T_{\gamma} P_{a b}\right\| \leqslant\left\|P_{a} T_{\gamma} P_{a}\right\|+\left\|P_{a b} T_{\gamma} P_{a}\right\|+\left\|Q_{b} T_{\gamma}\right\| .
$$

Let us show that the right hand side in (19) tends to zero as $a \rightarrow 0$ and $b \rightarrow \infty$, then the operator $T_{\gamma}$ will be compact from $L_{p}$ into $L_{q, u}$ as the uniform limit of compact operator ([11], VI.12).

Let $u_{a}=P_{a} u$, then by Theorem A we have

$$
\left\|P_{a} T_{\gamma} P_{a} f\right\|_{q, u_{a}} \leqslant\left\|P_{a} T_{\gamma} f\right\|_{q, u_{a}}=\left(\int_{0}^{\infty} u_{a}(x)\left|\int_{0}^{x} s^{\gamma-1} \ln \frac{x}{x-s} f(s) d s\right|^{q} d x\right)^{\frac{1}{q}} \ll
$$




$$
\ll \sup _{z>0} z^{\gamma+\frac{1}{p^{\prime}}}\left(\int_{z}^{\infty} u_{a}(x) x^{-q} d x\right)^{\frac{1}{q}}\|f\|_{p}
$$

Thus,

$$
\begin{gathered}
\left\|P_{a} T_{\gamma} P_{a}\right\| \ll \sup _{z>0} z^{\gamma+\frac{1}{p^{\prime}}}\left(\int_{z}^{\infty} u_{a}(x) x^{-q} d x\right)^{\frac{1}{q}}=\sup _{0<z<a} z^{\gamma+\frac{1}{p^{\prime}}}\left(\int_{z}^{a} u(x) x^{-q} d x\right)^{\frac{1}{q}} \leqslant \\
\leqslant \sup _{0<z<a} z^{\gamma+\frac{1}{p^{\prime}}}\left(\int_{z}^{\infty} u(x) x^{-q} d x\right)^{\frac{1}{q}}=\sup _{0<z<a} D_{\gamma}(z) .
\end{gathered}
$$

It yields

$$
\lim _{a \rightarrow 0}\left\|P_{a} T_{\gamma} P_{a}\right\| \ll \varlimsup_{z \rightarrow 0} D_{\gamma}(z)=\lim _{z \rightarrow 0} D_{\gamma}(z)=0 .
$$

The estimate $\left\|P_{a b} T_{\gamma} P_{a}\right\|$ for is

$$
\begin{gathered}
\left\|P_{a b} T_{\gamma} P_{a} f\right\|_{q, u}=\left(\int_{a}^{b} u(x)\left|\int_{0}^{x} s^{\gamma-1} \ln \frac{x}{x-s}\left(P_{a} f\right)(s) d s\right|^{q} d x\right)^{\frac{1}{q}} \leqslant \\
\leqslant\left(\int_{a}^{\infty} u(x)\left(\int_{0}^{a} s^{\gamma-1} \ln \frac{x}{x-s}|f(s)| d s\right)^{q} d x\right)^{\frac{1}{q}} \leqslant
\end{gathered}
$$

(we employ Hölder inequality and the properties of the function $x \ln \frac{x}{x-s}$ )

$$
\begin{gathered}
\leqslant\left(\int_{a}^{\infty} u(x)\left(\int_{0}^{a}\left|s^{\gamma-1} \ln \frac{x}{x-s}\right|^{p^{\prime}} d s\right)^{\frac{q}{p^{\prime}}} d x\right)^{\frac{1}{q}}\left(\int_{0}^{a}|f(s)|^{p} d s\right)^{\frac{1}{p}} \leqslant \\
\leqslant\left(\int_{a}^{\infty} u(x) x^{-q}\left(\int_{0}^{a}\left|s^{\gamma-1} x \ln \frac{x}{x-s}\right|^{p^{\prime}} d s\right)^{\frac{q}{p^{\prime}}} d x\right)^{\frac{1}{q}}\|f\|_{p} \leqslant \\
\leqslant\left(\int_{a}^{\infty} u(x) x^{-q} d x\right)^{\frac{1}{q}}\left(\int_{0}^{a}\left|s^{\gamma-1} a \ln \frac{a}{a-s}\right|^{p^{\prime}} d s\right)^{\frac{1}{p^{\prime}}}\|f\|_{p} \leqslant \\
\leqslant\left(\beta_{p}\right)^{\frac{1}{p^{\prime}}} a^{\gamma+\frac{1}{p^{\prime}}}\left(\int_{a}^{\infty} u(x) x^{-q} d x\right)^{\frac{1}{q}}\|f\|_{p} \leqslant\left(\beta_{p}\right)^{\frac{1}{p^{\prime}}} D_{\gamma}(a)\|f\|_{p},
\end{gathered}
$$

where $\beta_{p}=\int_{0}^{1}\left|s^{\gamma-1} \ln \frac{1}{1-s}\right|^{p^{\prime}} d s \leqslant \ln ^{p^{\prime}} 2 \int_{0}^{\frac{1}{2}} s^{p^{\prime}(\gamma-1)} d s+\max \left\{1,2^{-p^{\prime}(\gamma-1)}\right\} \int_{\ln 2}^{\infty} t^{p^{\prime}} e^{-t} d t$. Hence, $\left\|P_{a b} T_{\gamma} P_{a}\right\| \ll D_{\gamma}(a)$. Therefore,

$$
\lim _{b \rightarrow \infty} \lim _{a \rightarrow 0}\left\|P_{a b} T_{\gamma} P_{a}\right\| \ll \lim _{a \rightarrow 0} D_{\gamma}(a)=0 .
$$


Let $u_{b}=Q_{b} u$, then due to Theorem A we get

$$
\begin{aligned}
\left\|Q_{b} T_{\gamma} f\right\|_{q, u} & =\left(\int_{0}^{\infty} u_{b}(x)\left|\int_{0}^{x} s^{\gamma-1} \ln \frac{x}{x-s} f(s) d s\right|^{q} d x\right)^{\frac{1}{q}} \ll \\
& \ll \sup _{z>0} z^{\gamma+\frac{1}{p^{\prime}}}\left(\int_{z}^{\infty} u_{b}(x) x^{-q} d x\right)^{\frac{1}{q}}\|f\|_{p} .
\end{aligned}
$$

It follows

$$
\begin{aligned}
& \left\|Q_{b} T_{\gamma}\right\| \ll \sup _{z>0} z^{\gamma+\frac{1}{p^{\prime}}}\left(\int_{z}^{\infty} u_{b}(x) x^{-q} d x\right)^{\frac{1}{q}}= \\
& =\sup _{z \geq b} z^{\gamma+\frac{1}{p^{\prime}}}\left(\int_{z}^{\infty} u(x) x^{-q} d x\right)^{\frac{1}{q}}=\sup _{z \geq b} D_{\gamma}(z) .
\end{aligned}
$$

Therefore,

$$
\lim _{b \rightarrow \infty}\left\|Q_{b} T_{\gamma}\right\| \ll \varlimsup_{z \rightarrow \infty} D_{\gamma}(z)=\lim _{z \rightarrow \infty} D_{\gamma}(z)=0 .
$$

It follows from (19), (20), (21), and (22) that the right hand side of (19) tends to zero as $a \rightarrow 0$ and $b \rightarrow \infty$. Theorem 1 is proven.

Passing to the adjoint operator and applying Theorem 1, we obtain

Theorem 2. Let $1<p \leqslant q<\infty, \gamma>1-\frac{1}{q}$. Then operator (8) is compact from $L_{p, v}$ into $L_{q}$ if and only if

$$
D_{\gamma}^{*}<\infty, \text { and } \lim _{x \rightarrow 0} D_{\gamma}^{*}(x)=\lim _{x \rightarrow \infty} D_{\gamma}^{*}(x)=0
$$

From Theorem 1 we immediately obtain

Theorem 3. Let $1<p \leqslant q<\infty$ and $v(x)=x^{-\gamma}$. Fractional integration operator of infinitesimal order (4) is compact from $L_{p, v}$ into $L_{q, u}$ if and only if $D_{\gamma}<\infty$ and (9) holds true.

In the case $q<p$ we have

Theorem 4. Let $1<q<p<\infty, v(x)=x^{-\gamma}, \gamma>\frac{1}{p}$. Fractional integration operator of infinitesimal order (4) is compact from $L_{p, v}$ in $L_{q, u}$ if and only if

$$
E_{\gamma}=\left(\int_{0}^{\infty}\left[\left(\int_{x}^{\infty} \frac{u(t)}{t^{q}} d t\right)^{\frac{1}{q}} x^{\gamma+\frac{1}{p^{\prime}}}\right]^{\frac{p q}{p-q}} d x\right)^{\frac{p-q}{p q}}<\infty .
$$

Theorem 2 follows directly from Theorem 2 in work [9], since by Ando theorem ([12], $\S 5)$ for $1<q<p<\infty$ each bounded integral operator from $L_{p}$ in $L_{q, u}$ is compact. 


\section{BIBLIOGRAPHY}

1. E.M. Dyn'kin, B.P. Osilenker Weighted estimates of singular integrals and their applications // Matem. analiz. V. 21 (Itogi nauki i tekhniki. VINITI AN SSSR). M. 1983, P. 42-129. [ J. Soviet Math. 1985. V. 30, No. 3. P. 2094-2154.]

2. A. Kufner, L-E. Persson. Weighted inequalities of Hardy type. World Scientific, New Jersey, 2003.

3. R. Oinarov. Boundedness and compactness of Volterra type integral operators // Sibir. matem. zhurn. 2007. V. 48, No. 5. P. 1100-1115. [ Siberian Math. J. 2008. V. 48, No. 5. P. 884-896.]

4. K.F. Andersen, E.T. Sawyer. Weighted norm inegualities for the Riemann - Liouville and Weyl fractional integral operators// rans. Amer. Math. Soc. 1988, V. 308, 2. . 547-557.

5. D.V. Prokhorov. On the boundedness and compactness of a class of integral operators // J.London Math. Soc. 2000. V. 61, 2. . 617-628.

6. A. Meskhi. Solution of some weight problems for the Riemann - Liouville and Weil operators // Georgian Math.J. 1998. 5. P. 565-574.

7. D.V. Prokhorov, V.D. Stepanov. On Riemann-Liouville operators // Doklady RAN. 2002, V. 382, No. 4. P. 452-455. [Dokl. Math. 2002. V. 65, No. 1, P. 64-76.]

8. A.M. Nakhushev. Equations of mathematical biology. M.: Vysshaya shkola, 1995. (in Russian).

9. A.M. Abylaeva, M.Zh. Omirbek. Weighted estimate for integral operator with a logarithmic singularity // Izvestia, ser. fiziko-matem. Almaty: NAN RK. 2005, No. 1, P. 38-47. (in Russian).

10. L.V. Kantorovich, G.P. Akilov. Functional analysis. M.: Nauka, 1977 [Pergamon Press, 1982.]

11. M. Reed, B. Simon. Methods of modern mathematical physics. V. 1. Academic Press, N.Y. 1980.

12. M.A. Krasnoselskii, P.P. Zabreiko, E.I. Pustylnik, P.E. Sobolevskii. Integral operators in spaces of summable functions. M.: Nauka. 1966. [Noordhoff International Publishing, Leyden. 1976.]

Abylaeva Akbota Mukhamediyarovna,

L.N. Gumilyov Eurasian National University,

Munaitpasov str., 5

473021, Astana, Kazakhstan

E-mail: abylayeva_b@mail.ru

Baiarystanov Askar Ojnarovich,

L.N. Gumilyov Eurasian National University,

Munaitpasov str., 5,

473021, Astana, Kazakhstan

E-mail: oskar_62@mail.ru 